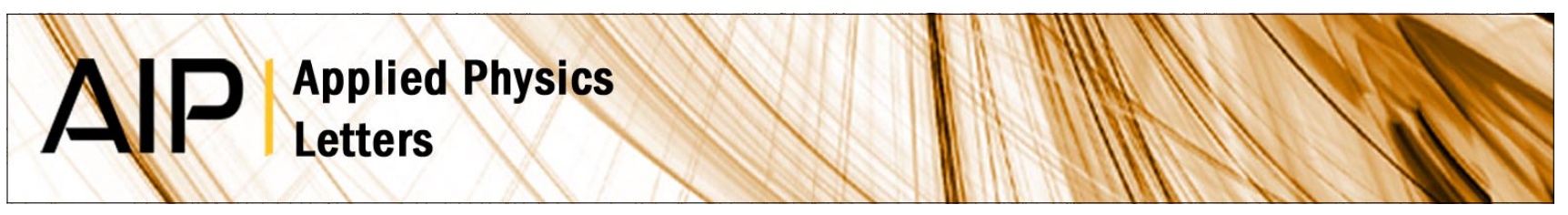

\title{
Tunable coupled surface acoustic cavities
}

M. M. de Lima, P. V. Santos, Yu. A. Kosevich, and A. Cantarero

Citation: Appl. Phys. Lett. 100, 261904 (2012); doi: 10.1063/1.4730398

View online: http://dx.doi.org/10.1063/1.4730398

View Table of Contents: http://apl.aip.org/resource/1/APPLAB/v100/i26

Published by the American Institute of Physics.

\section{Related Articles}

Extremely low-loss acoustic phonons in a quartz bulk acoustic wave resonator at millikelvin temperature Appl. Phys. Lett. 100, 243504 (2012)

Extraction of second order piezoelectric parameters in bulk acoustic wave resonators Appl. Phys. Lett. 100, 232901 (2012)

Acoustic resonator based on periodically poled transducers: Concept and analysis J. Appl. Phys. 111, 064106 (2012)

Viscosity sensor using $\mathrm{ZnO}$ and AIN thin film bulk acoustic resonators with tilted polar c-axis orientations J. Appl. Phys. 110, 094511 (2011)

Correlations between microstructure and Q-factor of tunable thin film bulk acoustic wave resonators J. Appl. Phys. 110, 054102 (2011)

\section{Additional information on Appl. Phys. Lett.}

Journal Homepage: http://apl.aip.org/

Journal Information: http://apl.aip.org/about/about_the_journal

Top downloads: http://apl.aip.org/features/most_downloaded

Information for Authors: http://apl.aip.org/authors

\section{ADVERTISEMENT}

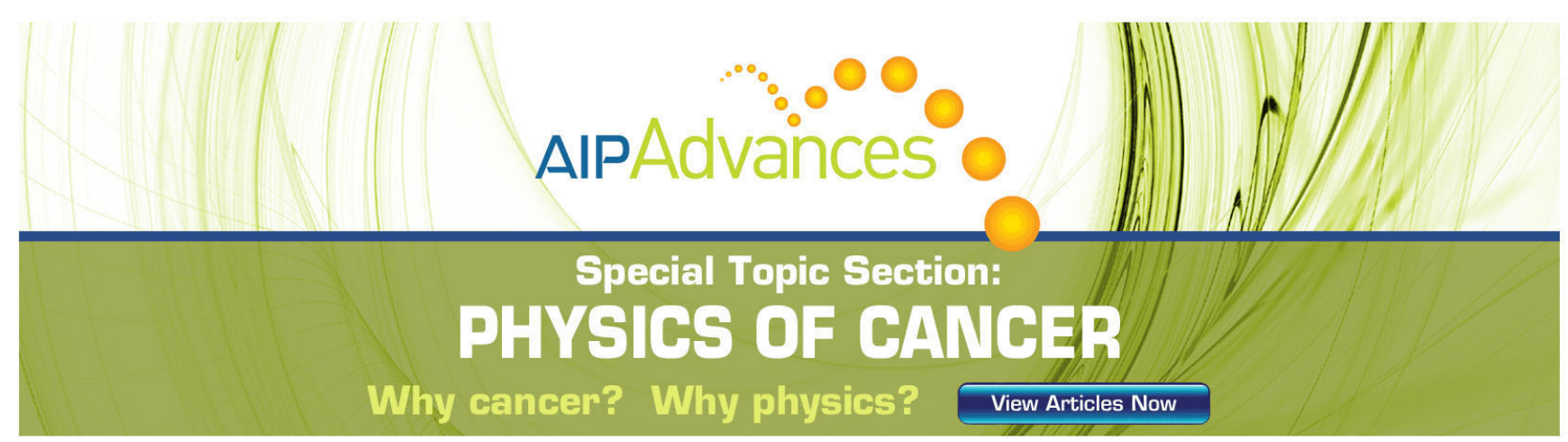




\title{
Tunable coupled surface acoustic cavities
}

\author{
M. M. de Lima, Jr., ${ }^{1,2, a)}$ P. V. Santos, ${ }^{3}$ Yu. A. Kosevich, ${ }^{1,4}$ and A. Cantarero ${ }^{1}$ \\ ${ }^{1}$ Materials Science Institute, Universitat de València, P. O. Box 22085, E-46071 Valencia, Spain \\ ${ }^{2}$ Fundaciò General de la Universitat de València, E-46010 Valencia, Spain \\ ${ }^{3}$ Paul-Drude-Institut für Festkörperelektronik, Hausvogteiplatz 5-7, D-10117 Berlin, Germany \\ ${ }^{4}$ Semenov Institute of Chemical Physics, Russian Academy of Sciences, ul. Kosygina 4, \\ 119991 Moscow, Russia
}

(Received 2 March 2012; accepted 6 June 2012; published online 26 June 2012)

\begin{abstract}
We demonstrate the electric tuning of the acoustic field in acoustic microcavities (MCs) defined by a periodic arrangement of metal stripes within a surface acoustic delay line on $\mathrm{LiNbO}_{3}$ substrate. Interferometric measurements show the enhancement of the acoustic field distribution within a single MC, the presence of a "bonding" and "anti-bonding" modes for two strongly coupled MCs, as well as the positive dispersion of the "mini-bands" formed by five coupled MCs. The frequency and amplitude of the resonances can be controlled by the potential applied to the metal stripes. (C) 2012 American Institute of Physics. [http://dx.doi.org/10.1063/1.4730398]
\end{abstract}

The semiconductor superlattices ${ }^{1}$ enables the ad hoc design of electronic wave functions in a solid by allowing the fabrication of complex architectures in which the coupling between electronic states can be externally tuned using an external bias. Perhaps one of the most striking examples of this concept are quantum cascade lasers, ${ }^{2}$ whose sophisticated wave function engineering leads to light amplification through intersubband transitions. The exploitation of structural modification for coupling control has also been extended to other solid-state elementary excitations. Examples are photonic "molecules" based on photonic cavities ${ }^{3}$ or $\mathrm{THz}$ acoustic amplification by stimulated emission in biased phononic superlattices. ${ }^{4}$ In the case of surface acoustic phonons, we have recently demonstrated the presence of Wannier-Stark ladders and Landau-Zener tunneling in coupled phononic cavities. ${ }^{5}$ In this Letter, we take advantage of the planar geometry of this approach to perform a detailed investigation of the acoustic field distribution of coupled acoustic microcavities (MCs) by means of interferometry. We then show that the acoustic field distribution can be electrically tunned by controlling the potential of the $\mathrm{MC}$ electrodes.

The experiments were carried out using surface acoustic wave (SAW) delay lines on a $128^{\circ}$-rotated Y-cut $\mathrm{LiNbO}_{3}$ substrate [see Fig. 1(a)]. The split-finger aluminum interdigital transducers (IDTs) of the delay lines were designed for an acoustic wavelength $\lambda=11.2 \mu \mathrm{m}$, which corresponds to a center resonance frequency of approximately $f_{\text {SAW }} \approx 340 \mathrm{MHz}$. The coupled MCs were fabricated by depositing NiCr/Au gratings with thicknesses $h=10 / 110 \mathrm{~nm}$ within the SAW delay lines. The acoustic contrast between the metal-free and metal-coated region results from the mechanical loading as well as the screening of the piezoelectric potential underneath the metal stripes. We have investigated four different grating structures: a bare Bragg reflector (BR), a single cavity (SC), two coupled cavities (2C), and five coupled cavities (5C). Each BR consists of $20 \lambda / 4$ stripes (corresponding to a width of $2.8 \mu \mathrm{m}$ ) separated by a $\lambda / 4$

a)mmlimajr@uv.es. free-surface $\mathrm{LiNbO}_{3}$ spacing. The bare $\mathrm{BR}$ investigated is 6-fold longer than the individual BRs used to define the MCs [cf. Fig. 1(a)]. In all MCs, the spacers in-between the two BRs were nominally $3 \lambda / 2$ wide. The metallic stripes could be either electrically isolated from each other or shortcircuited all together in order to control their acoustic properties.

The solid lines in Fig. 2 compare the acoustic transmission through the different structures with floating (a) and short-circuited (b) stripes measured using a network analyzer. The BR introduces a transmission stop band in Fig. 2 while the SC creates a transmission resonance within this stop band. The strong coupling in the double MCs (2C) leads to the appearance of two transmission resonances at low frequency (LF) and high frequency (HF). ${ }^{6}$ The structure with 5 cavities (5C) displays five resonances within the stop band with frequency separation much smaller than for the $2 \mathrm{C}$. Due to the periodicity and the strong coupling between the

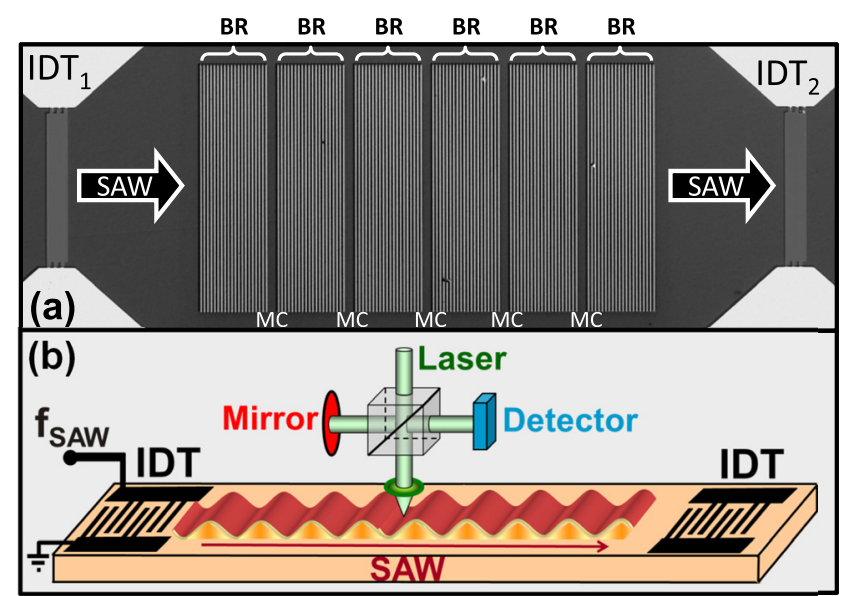

FIG. 1. (a) Micrograph of a sample with five coupled microcavities (5C) defined by metal stripes forming Bragg reflectors (BRs). The SAWs are excited by the interdigital transducer IDT $_{1}$ and detect after propagation through the MCs by IDT $_{2}$. (b) Michelson interferometer used to probe the time-averaged squared amplitude of the vertical particle displacement $\left(\left\langle u_{z}^{\prime 2}\right\rangle\right)$ along the surface of the sample. 

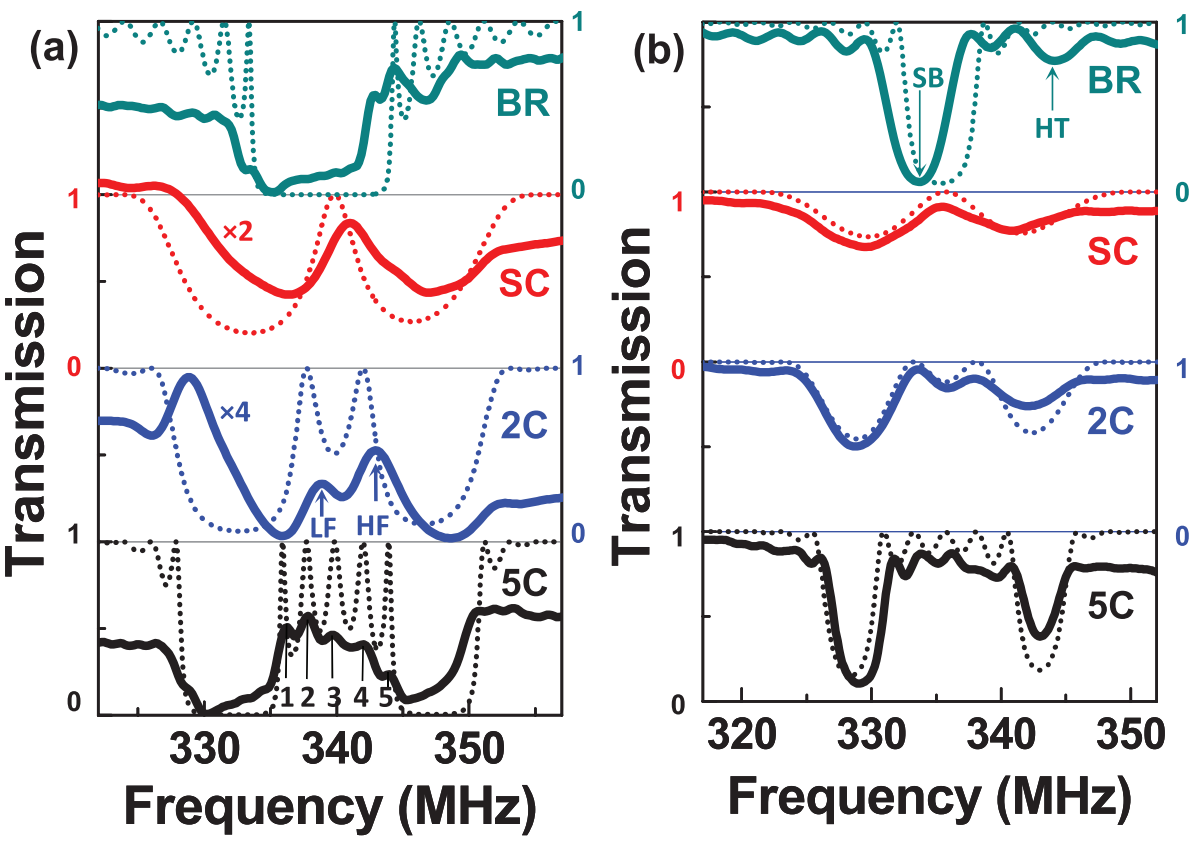

FIG. 2. Experimental (solid lines) and calculated (dotted lines) transmission spectra for a set of grating structures with floating (a) and short-circuited (b) stripes. From top to bottom, they correspond to a 120-stripes BR, a SC, two coupled cavities (2C), and five coupled cavities (5C). SB and HT stand for stop-band and high transmission, respectively. LF and HF correspond to upper and lower double cavity resonance frequency, respectively. The numbers from 1 to 5 indicate the order of the Fabry-Perot modes. cavities, these resonances form a mini-band with frequency separation smaller than their individual width.

The acoustic contrast between the metal-coated and metal-free regions on a $\mathrm{LiNbO}_{3}$ substrate has been discussed in detail in Ref. 7 and depends on the mechanical loading introduced by the stripe (this includes the mass and stress loading effects) as well as on the piezoelectric screening due to the metal coating for shorted-circuit gratings. For strong piezoelectric materials such as the case of $\mathrm{LiNbO}_{3}$, the reflection coefficient under floating condition (for the ratio $h / \lambda \approx 0.01$ ) displays the opposite phase and is larger than the one for short-circuit configuration. These effects are readily seen by comparing the transmission for floating and shorted MCs in Figs. 2(a) and 2(b), respectively. In the latter, the width of the stop band reduces due to the lower contrast while the frequency of the resonances decreases by approximately $1.6 \%$. These results demonstrate the electrical tuning of the acoustic response of the coupled cavities.

The dashed lines in Fig. 2 display transmission spectra calculated using the model described in Ref. 5. In the calculations, we assumed the reflection coefficient of shortcircuited stripes to be reduced by $50 \%$ in comparison to the ones for floating stripes discussed in that work. In all cases, reasonable coincidence is observed between theory and experiments, with an overall excellent agreement for the short-circuited case. The larger attenuation presented by the floating electrodes is attributed to coupling to higher frequencies shear modes within the gratings. ${ }^{8}$

A major advantage of the MC planar geometry is the possibility of accessing the acoustic field along the structures. Figure 1(b) sketches the microscopic Michelson interferometer used to that purpose. Here, the reflection of a $532 \mathrm{~nm}$ single-mode laser beam focused onto an approximately $1-\mu \mathrm{m}$ spot on the surface of the sample interferes with a reference beam at a fast photodiode. By means of a spectrum analyzer, we detect the photodiode signal $\left\langle u_{z}^{\prime 2}\right\rangle$ at the first side band of the SAW frequency, which is proportional to the time-averaged squared amplitude of the vertical particle displacement $\left\langle u_{z}^{2}\right\rangle$ modified by the optical reflection of the metal stripes. Spatial profiles were obtained by scanning the laser beam along the surface of the samples. A Rayleigh SAW induces both vertical and horizontal vibrations of the surface. Interferometric measurements on a flat surface normally probe the vertical surface displacement, which oscillates at the SAW frequency $f_{\text {SAW }}$ and increases with the surface reflectivity. In the stripe regions, however, the interfaces between the metal-coated and metal-free regions induce an additional contribution to the interferometric signal at the same frequency $f_{\mathrm{SAW}}$, which cannot be disentangled from the vertical motion. In the calculated profiles, we have convoluted the calculated field distribution using the method described in Ref. 5 with the static reflectivity profile of the gratings before comparing the simulations with the experiments. These profiles, however, do not take into account the effects of the in-plane motion induced by the SAW.

Figure 3(a) compares interferometric profiles for the bare BR measured within the transmission stop band (SB) and obtained at a high transmission (HT) frequency, as indicated by the arrows in Fig. 2(b). The dash-dotted vertical lines indicate the edges of the BR grating and separate the profiles in three regions. The center portion comprises the grating itself while the left and right sides correspond to the region closer to the source (IDT ${ }_{1}$ in Fig. 1) and detector $\left(\mathrm{IDT}_{2}\right)$. Outside the stop band, $\left\langle u_{z}^{\prime 2}\right\rangle$ is approximately constant at both sides of the BR, thus indicating a high SAW transmission through the structure. Within the central region, the formation of a constant-amplitude standing wave is easily demonstrated by the presence of nodes in $\left\langle u_{z}^{\prime 2}\right\rangle$ with $\lambda / 2$ periodicity. The apparent increase in $\left\langle u_{z}^{\prime 2}\right\rangle$ within the BR is an experimental feature caused by the higher reflectivity of the metal stripes relative to the bare $\mathrm{LiNbO}_{3}$ surface. In contrast, when the excitation frequency is at the center of the stop band [SB in the upper panel of Fig. 3(a)], $\left\langle u_{z}^{\prime 2}\right\rangle$ decays exponentially within the BR and the transmission vanishes. Furthermore, a standing wave forms in the region between the source IDT and the BR due to the high grating reflectivity at this frequency. 

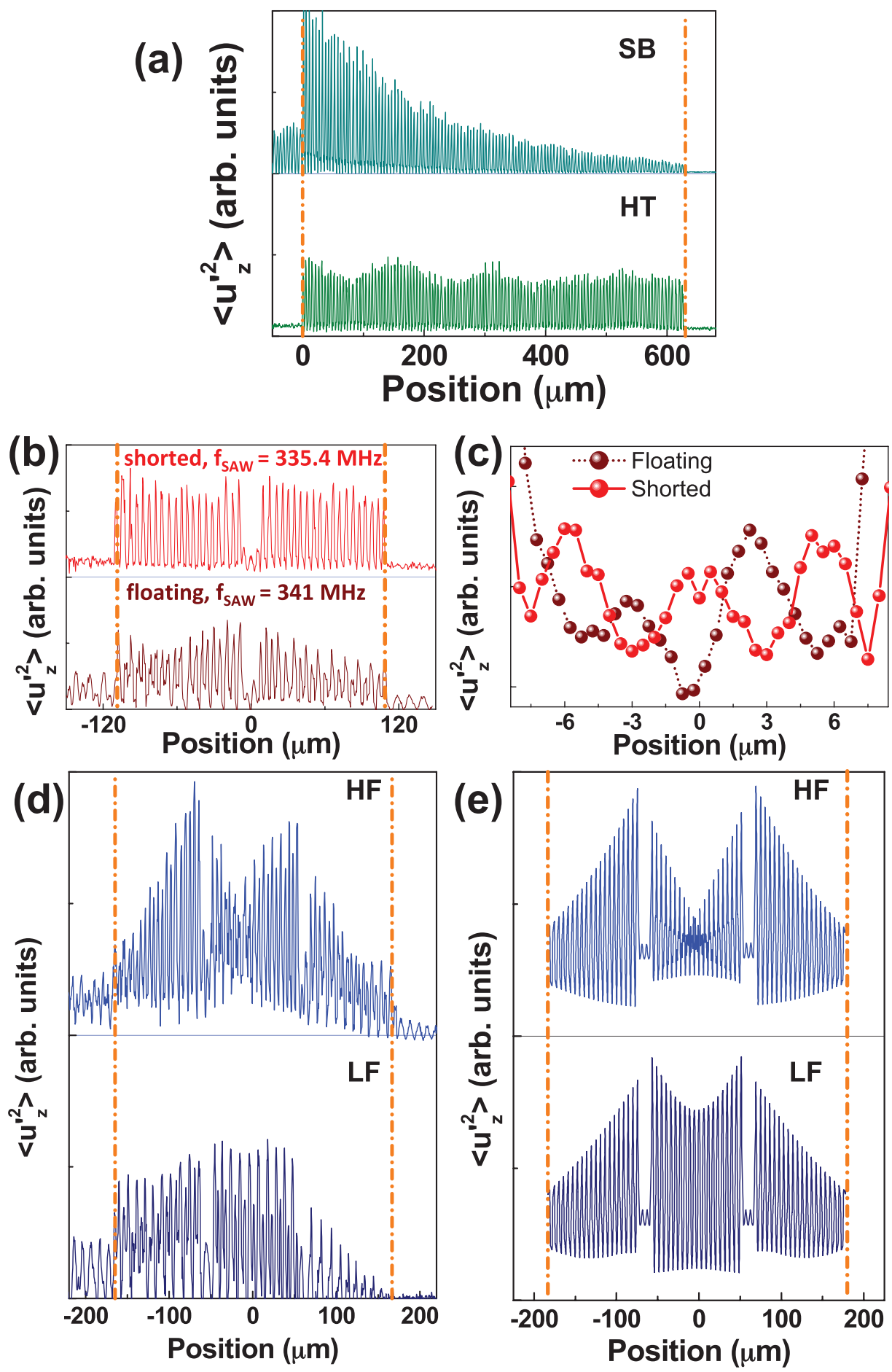

FIG. 3. (a) Measured field $\left\langle u_{z}^{2}\right\rangle$ for a BR with shorted stripes at the excitation frequencies SB and HT indicated in Fig. 2(b). The vertical dash-dotted lines comprise the region defined by the grating. (b) Measured $\left\langle u_{z}^{\prime 2}\right\rangle$ at the resonance transmission frequency of the SC for floating (lower panel) and shorted stripes (upper panel). (c) Blowup of the results in (b) within the MC spacer layer. (d) and (e) display, respectively, the measurements and calculations of $\left\langle u_{z}^{\prime 2}\right\rangle$ for the $2 \mathrm{C}$ structure with floating stripes at the frequencies LF and HF indicated in Fig. 2.
Figure 3(b) shows $\left\langle u_{z}^{\prime 2}\right\rangle$ for the SC with floating (lower panel) and shorted stripes (upper panel). The reduction in acoustic impedance contrast induced by short-circuiting the stripes has pronounced effects on the field distribution. First, the enhancement of the acoustic fields around the MC spacer is less pronounced for the short-circuited MC than for the floating one. The increase of the envelop of the acoustic field due to confinement in the central region of the structure is clearly observed in the case of the floating grating. As mentioned before, the smaller signal at the cavity region is due to the low reflectivity of the $\mathrm{LiNbO}_{3}$ surface. Second, there are significant changes in the standing wave field pattern within the spacer, as demonstrated by the zoomed profiles of Fig. 3(c). Here, the node observed at the center of the spacer for floating electrodes turns into an anti-node when the stripes are short-circuited. The latter is attributed to the change in the sign of the acoustic reflection coefficient when the gratings change from floating to short-circuit configuration.

In the case of two nominally identical cavities with floating electrodes, the field distributions for both LF and HF modes are shown in Fig. 3(d) for floating cavities. These modes have different symmetries. Both cavities vibrate in phase for the LF mode, leading to a field maximum inbetween the cavities ("bonding" mode). For the HF mode, in contrast, the two cavities vibrate out-of-phase, thus creating a field node in-between them ("anti-bonding" mode). For 

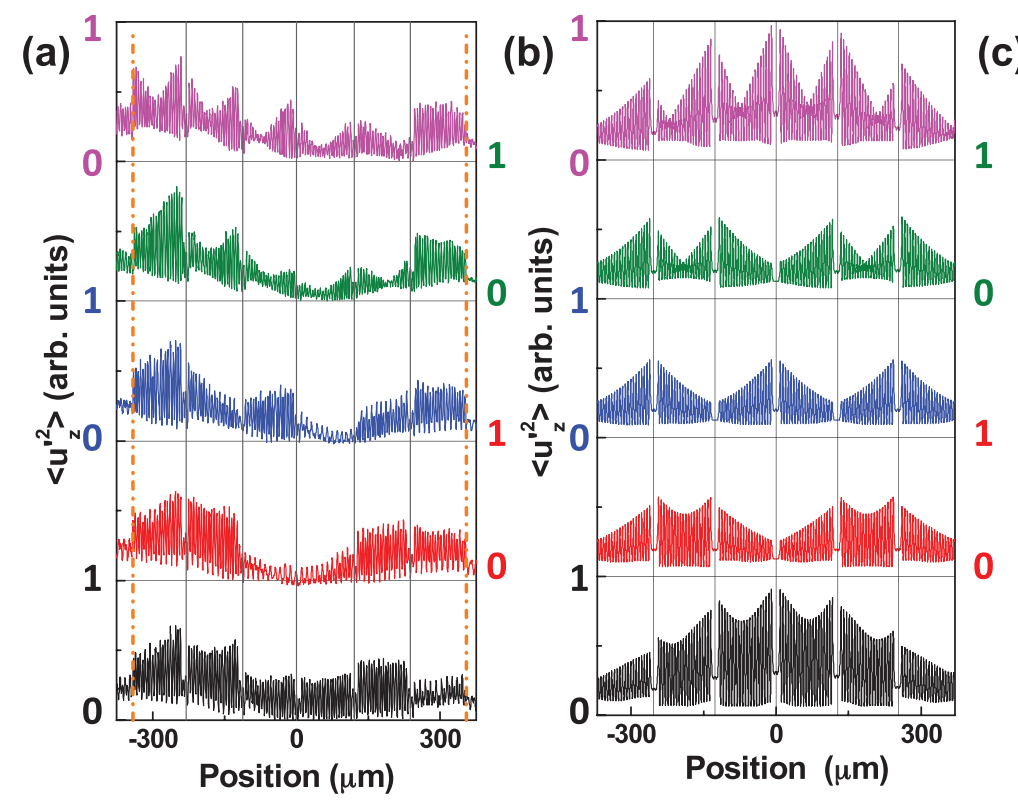

(c)

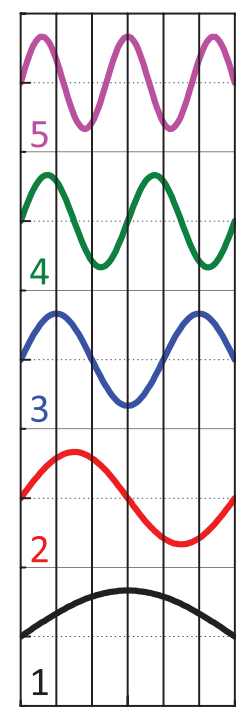

FIG. 4. (a) Measured and (b) calculated $\left\langle u_{z}^{\prime 2}\right\rangle$ profiles for five coupled MCs (5C) with floating stripes for the excitation frequencies 1 to 5 (from bottom to top) indicated in the lowest panel of Fig. 2(a). The vertical dash-dotted lines indicate the edges of the structure while the vertical solid lines mark the position of the MCs spacers. (c) Schematic diagram illustrating the expected field envelops for minibands with positive dispersion. both frequencies, the measured profiles are well-reproduced by convoluting the calculated field profiles with the optical reflectivity of the $\mathrm{NiCr} / \mathrm{Au}$ and bare- $\mathrm{LiNbO}_{3}$ regions, which are shown in Fig. 3(e).

Finally, Figs. 4(a) and 4(b) compares measured and calculated $\left\langle u_{z}^{\prime 2}\right\rangle$ profiles, respectively, for five coupled MCs with floating electrodes. They were obtained for excitation frequencies corresponding to the 5 maxima of the FabryPerot oscillations in the transmission experiments of Fig. 2(a). The evolution of the field distribution demonstrates the positive dispersion of the transmission mini-band investigated. In other words, the eigenfunction of the lowest eigenfrequency corresponds to a mode with the smallest wavevector, as it is sketched in Fig. 4(c) for the envelop of $u_{z}$.

In summary, we have shown that the field distribution as well as the acoustic transmittance band of surface acoustic MCs on $\mathrm{LiNbO}_{3}$ depend on whether the metal stripes are floating or electrically shorted, thus, demonstrating that these quantities can be electrically controlled by varying the impedance between the stripes. Measurements of the acoustic field by interferometry shows field confinement in a single
MC, "bonding" and "anti-bonding" modes for two coupled MCs, as well as the positive dispersion of a "mini-band" formed by five coupled MCs. The resonance frequency and the coupling strength can be controlled by changing the potential of the MC stripes.

M.M.L. thanks the Spanish Ministry of Science and Innovation (Grant No. TEC2009-12075) for financial support.

${ }^{1}$ L. Esaki and R. Tsu, IBM J. Res. Dev. 14, 61 (1970).

${ }^{2}$ J. Faist, F. Capasso, D. L. Sivco, C. Sirtori, A. L. Hutchinson, and A. Y. Cho, Science 264, 553 (1994).

${ }^{3}$ M. Bayer, T. Gutbrod, J. P. Reithmaier, A. Forchel, T. L. Reinecke, P. A. Knipp, A. A. Dremin, and V. D. Kulakovskii, Phys. Rev. Lett. 81, 2582 (1998).

${ }^{4}$ R. P. Beardsley, A. V. Akimov, M. Henini, and A. J. Kent, Phys. Rev. Lett. 104, 085501 (2010).

${ }^{5}$ M. M. de Lima, Jr., Yu. A. Kosevich, P. V. Santos, and A. Cantarero, Phys. Rev. Lett. 104, 165502 (2010).

${ }^{6}$ I. D. Avramov, A. Voigt, and M. Rapp, Electron. Lett. 41, 450 (2005).

${ }^{7}$ D.-P. Chen and H. A. Haus, IEEE Trans. Sonics Ultrason. 32, 395 (1985).

${ }^{8}$ A. P. Mayer, W. Zierau, and A. A. Maradudin, J. Appl. Phys. 69, 1942 (1991). 